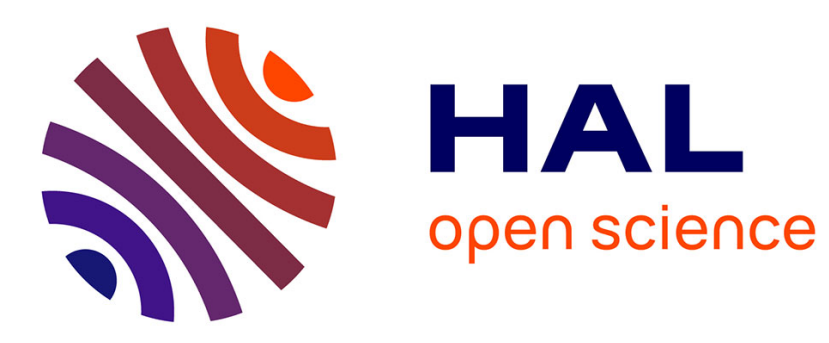

\title{
Ultracompact silicon-on-insulator ridge-waveguide mirrors with high reflectance
}

Philippe Velha, Jean-Claude Rodier, Philippe Lalanne, Jean-Paul Hugonin, David Peyrade, Eric Picard, Thomas Charvolin, Emmanuel Hadji

\section{- To cite this version:}

Philippe Velha, Jean-Claude Rodier, Philippe Lalanne, Jean-Paul Hugonin, David Peyrade, et al.. Ultracompact silicon-on-insulator ridge-waveguide mirrors with high reflectance. Applied Physics Letters, 2006, 89 (17), pp.171121. 10.1063/1.2372581 . hal-00394756

\section{HAL Id: hal-00394756 https://hal.science/hal-00394756}

Submitted on 21 Oct 2013

HAL is a multi-disciplinary open access archive for the deposit and dissemination of scientific research documents, whether they are published or not. The documents may come from teaching and research institutions in France or abroad, or from public or private research centers.
L'archive ouverte pluridisciplinaire HAL, est destinée au dépôt et à la diffusion de documents scientifiques de niveau recherche, publiés ou non, émanant des établissements d'enseignement et de recherche français ou étrangers, des laboratoires publics ou privés. 


\title{
Ultracompact silicon-on-insulator ridge-waveguide mirrors with high reflectance
}

\author{
P. Velha, ${ }^{\text {a) }}$ J. C. Rodier, P. Lalanne, ${ }^{\text {b) }}$ and J. P. Hugonin \\ Laboratoire Charles Fabry de l'Institut d'Optique, Centre National de la Recherche Scientifique, \\ Université Paris-Sud, Campus Polytechnique, RD 128, 91127 Palaiseau Cedex, France \\ D. Peyrade \\ Laboratoire des Technologies de la Microélectronique, Centre National de la Recherche Scientifique, \\ UMR5129, 17 rue des Martyrs, 38054 Grenoble Cedex 9, France \\ E. Picard, T. Charvolin, and E. Hadji \\ Laboratoire Silicium Nanoélectronique Photonique et Structure, Département de Recherche \\ Fondamentale sur la Matière Condensée, Commissariat à l'Energie Atomique, 17 rue des Martyrs, \\ F-38054 Grenoble Cedex, France
}

(Received 10 March 2006; accepted 20 September 2006; published online 27 October 2006)

\begin{abstract}
Microcavities offering small modal volumes $V \approx 0.6(\lambda / n)^{3}$ and consisting of two identical tapered Bragg mirrors etched into a monomode silicon-on-insulator ridge waveguide are studied for operation at telecommunications wavelengths. The authors have measured a $Q$ factor of 8900 , for a loaded cavity with a peak transmission at resonance in excess of $60 \%$. The measured $Q$ value quantitatively agrees with the calculation results and is 20 times larger than those previously reported for similar geometries without tapers. (C) 2006 American Institute of Physics.
\end{abstract}

[DOI: $10.1063 / 1.2372581]$

High- $Q$ optical microcavities, which confine light in wavelength-sized modal volumes $V$, are essential components of many optical devices and effects, from spontaneousemission inhibition or enhancement, ${ }^{1}$ threshold laser reduction, to a wide range of processing functions in integrated systems. ${ }^{2}$ Light confinement can be achieved by total internal reflection (TIR), Bragg reflection, or a combination of both mechanisms. In ring or disk resonators, confinement solely relies on TIR. These microcavities can be cascaded to implement various optical functions, ${ }^{3,4}$ and with strong lateral confinement as in silicon-on-insulator (SOI) substrates, the disk or the ring resonators can have a very small $(1-3 \mu \mathrm{m})$ diameter with small bending loss. ${ }^{2}$ Microcavities in twodimensional photonic-crystal (PC) slabs are also highly valuable candidates for achieving high $Q$ 's and small $V$ 's. Presently, $Q$ factors above $10^{5}$ with wavelength-sized $V$ 's are available in several research laboratories, see Refs. 5 and 6 and references therein. However, these recorded $Q$ values are obtained by etching off the sacrificial buried layer under the PC slab to form a suspended membrane in air. This puts severe restrictions on large-scale integration. Apparently, achieving high- $Q$ PC microcavities on a substrate is not obvious: as shown in a recent report, ${ }^{7} \mathrm{PC}$ cavities with $Q$ $=5000$ in a silicon membrane offer $Q$ values ten times smaller once implemented without removing the $\mathrm{SiO}_{2}$ layer.

The microcavities considered in this work are fabricated in a SOI substrate. For integrated photonics, silicon is an ideal material platform because of its maturity in the electronics industry, offering the possibility to combine both photonic and electronic devices all on one chip. Like ring resonators, a classical translation-invariant ridge waveguide is used to confine light in the transversal directions. The

\footnotetext{
${ }^{a)}$ Also at Laboratoire des Technologies de la Microélectronique and CEA Laboratory SiNaPS.

b)Electronic mail: philippe.lalanne@iota.u-psud.fr
}

longitudinal confinement is achieved by mirrors composed of subwavelength holes, like in PC cavities. In comparison with earlier works on similar geometries, ${ }^{8-10}$ the mirrors are not fully periodic. Through a fine geometry tuning designed with Bloch-mode-engineering concepts, ${ }^{11,12}$ they additionally incorporate a taper section composed of four holes, see Fig. 1. The taper section aims at reducing the transverse-modeprofile mismatch at the interface between the periodic section of the mirrors and the ridge waveguide, and thus at canceling out-of-plane radiation losses. A $Q$ factor of 8900 has been measured at midgap frequency $(\lambda=1.58 \mu \mathrm{m})$ for a loaded cavity with a peak transmission larger than $60 \%$. The measured $Q$ value represents a 20-fold improvement over previously reported $Q$ 's for similar in-line geometries ${ }^{8-10}$ or over three-hole-missing two dimensional photonic-crystal cavities recently fabricated on a $\mathrm{SiO}_{2}$ substrate. ${ }^{7}$

The cavity structures are fabricated from SOI wafers with a $340 \mathrm{~nm}$ single crystal $\mathrm{Si}$ layer on a $2 \mu \mathrm{m} \mathrm{SiO} \mathrm{S}_{2}$ layer. The ridge and hole patterning is performed by electron-beam lithography with a Leica VB-6UHR vector scan generator in a 400-nm-thick NEB22 negative resist spin coated on the

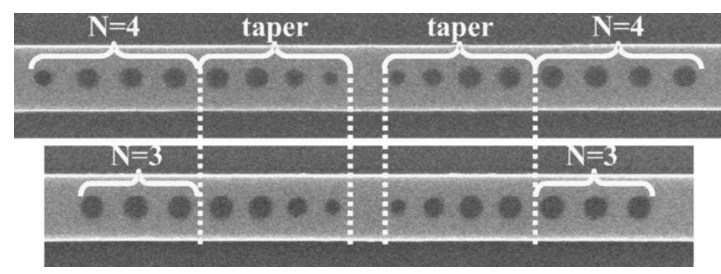

FIG. 1. SEM photographs of two microcavities considered in this work. The Bragg mirrors are composed of a four-hole taper and of a periodic section with $N$ holes ( $N=3$ and 4 in the photographs). The taper hole diameters, measured with the SEM, are $130,160,185$, and $181 \mathrm{~nm}$ with center-tocenter hole distances of $300,315,325$, and $352 \mathrm{~nm}$. The periodic section of the mirrors is composed of holes with a $181 \mathrm{~nm}$ diameter and the periodicity constant is $a=370 \mathrm{~nm}$. The side-to-side distance between the two inner holes (the physical length of the cavities) is $L=425 \mathrm{~nm}$. 
sample. After developing the resist, etching is performed in an inductively coupled industrial plasma chamber (DPS from Applied Materials Inc.). The silicon layer is then etched with a $\mathrm{Cl}_{2}, \mathrm{HBr}$, and $\mathrm{O}_{2}$ gas mixture, using the $\mathrm{SiO}_{2}$ layer as an etch stop. Finally, resist is stripped by oxygen plasma. For testing purposes, the wafer is manually cleaved to obtain optical facets. The lengths of the two access ridge waveguides are approximately 7 and $3.5 \mathrm{~mm}$, respectively. Several cavities with identical taper sections, but with a different number $(N=2,3$, and 4$)$ of holes in the periodic section, are fabricated on the same wafer. Because they are difficult to characterize with our in-line characterization setup, cavities with $N=5$ have not been fabricated. The layout also includes reference ridge waveguides (without cavities) for characterization purposes.

After fabrication, the cavities are first observed with a scanning electron microscope (SEM). Microcavity SEM photographs are shown in Fig. 1. The ridge width is $520 \mathrm{~nm}$ and typical hole diameters vary from 130 to $190 \mathrm{~nm}$. The wafer is also observed with an atomic force microscope (AFM), revealing that the surface roughness of the ridge sidewalls is $\approx 3 \mathrm{~nm} \mathrm{rms}$. The device under test is mounted on an, $X Y Z$ translational stage. TE-polarized light from a highresolution tunable external laser source $(1520-1620 \mathrm{~nm})$ is launched into the 7-mm-long access waveguide using a polarization-maintaining fiber connected to a microlensed fiber. The fiber tip produces a spot with a beam waist of $\approx 2.1 \mu \mathrm{m}$ with minimal mixing of TE-TM modes (rejection ratio of $20-30 \mathrm{~dB}$ ). After passing through the device, the light is collected by an imaging system composed of a $\times 20$ microscope objective (numerical aperture of 0.30) and of an antireflection coated planoconvex lens with a $50 \mathrm{~mm}$ focal length. The waveguide output is imaged either onto an IR camera for observing light at the output cleaved facet or onto an InGaAs photodiode for quantitative transmission measurements. Additionally, when using the InGaAs photodiode, the imaging system incorporates a $100 \mu \mathrm{m}$ pinhole and a polarizer in order to filter the inevitable spurious substrateguided light.

We first estimate the attenuation loss of the ridge waveguides. Five reference waveguides, without cavities, are characterized by measuring the contrast of the Fabry-Perot fringes resulting from the bouncing of light between the cleaved facets. Assuming a facet modal reflectivity of 0.36 , a value calculated with a fully vectorial three dimensional (3D) Fourier modal method, we obtain a straight-waveguideattenuation loss of $6 \pm 1 \mathrm{~dB} / \mathrm{cm}$. This value is compatible with the sidewall roughness measured with the AFM (Ref. 13) and is only twice larger that the best attenuation reported so far for similar SOI ridge geometries. ${ }^{14}$

The main results obtained for cavity-transmission measurements are summarized in Fig. 2. The dots in Fig. 2(a) represent the transmission spectrum recorded for the cavity with $N=4$. The data exhibit high-frequency oscillations, with large amplitudes at resonance. The oscillations can be easily interpreted by considering that the analyzed system is actually composed of three coupled cavities, the microcavity under study and two additional cavities formed by the cleaved facets and the microcavity itself. The solid curve in Fig. 2(b) shows the Fourier transform of the recorded spectrum. Three peaks at frequencies, labeled $\mathrm{f}_{1}, \mathrm{f}_{2}$, and $\mathrm{f}_{3}$ in the figure, are observed. These peaks also show up in the Fourier spectrum (dashed curve) of the cavity with $N=3$. The two first fre-
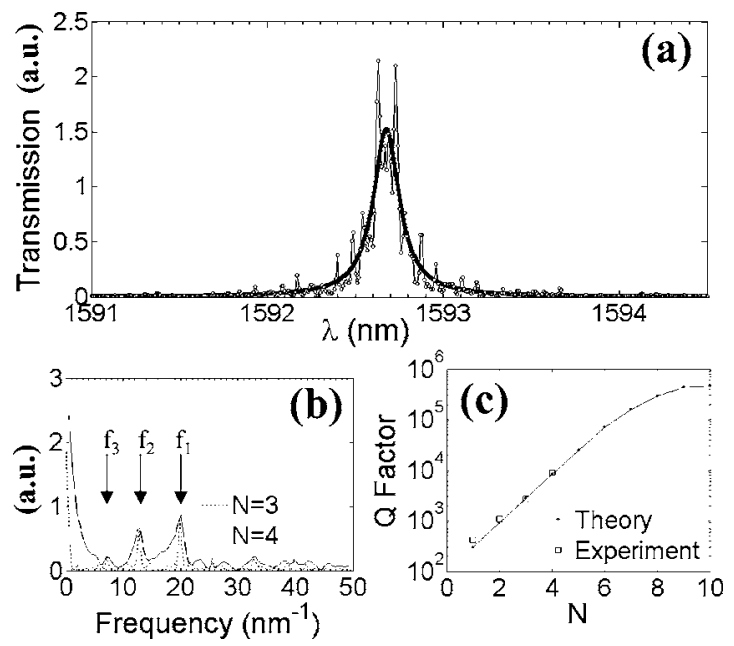

FIG. 2. Experimental results and data analysis. (a) Recorded transmission spectrum (in arbitrary units) of the microcavity with $N=4$ holes in the periodic section of the mirror. The high-frequency oscillations are Fabry-Perot fringes due to the bouncing of light between the cleaved facets. The solid bold curve is obtained after filtering. (b) Fourier transform of the cavity transmissions for the $N=4$ cavity (solid curve) and for the $N=3$ cavity (dashed curve). (c) Comparison between the measured and calculated $Q$ factors as a function of $N$. Squares: experimental data. Dots: $Q$ values obtained with the Fabry-Perot model from modal reflectivities $R_{N}$ calculated for geometric dimensions measured on the actual device.

quencies are due to the bouncing of light in the two access waveguides, and the third one represents the frequency difference $f_{1}-f_{2}$. As can be easily shown by analytic considerations, it is impossible to extract the $Q$ factor of the microcavity from the sole knowledge of the transmission of the coupled-cavity system. Additional information, such as the knowledge of the peak transmission $T_{\max }$ at resonance, is required. Nevertheless, to estimate the microcavity $Q$, we apply a low pass filter, filtering out all frequencies above $f_{3}$. After fitting to a Lorentzian function, we obtain the thick solid curve in Fig. 2(a), and a $Q$ factor of 8900 is inferred. Simulations performed with a $2 \times 2$ transfer-matrix approach have shown that the filtering technique generally underestimates the microcavity $Q$ factors. The relative difference may be as large as $20 \%$ for large Fabry-Perot oscillation amplitudes as those in Fig. 2(a). The same characterization procedure has been performed for microcavities with $N=2$ and 3 holes in the periodic sections of the mirrors. The measured $Q$ values are reported in Fig. 2(c) as squares.

In order to obtain more in-depth insight into the experimental results and the tapering effect, we have computed the modal reflectivities $R_{N}$ of the tapered mirrors for several values of the number $N$ of holes in the periodic section of the mirrors. The computations are performed with a 3D fully vectorial frequency-domain Fourier modal method ${ }^{15}$ for geometric dimensions measured on the actual device, see the caption of Fig. 1 for details. The thick curve in Fig. 3 shows $1-R_{\infty}$ over the 350-nm-wide mirror-band gap centered around $\lambda=1.55 \mu \mathrm{m}$. For semi-infinite mirrors $(N=\infty)$, $1-R_{\infty}$ represents the radiation loss in the cladding due to the mode-profile mismatch at the mirror interface. Because the SEM technique used to determine the hole diameters has a limited accuracy of $\pm 5 \mathrm{~nm}$, we have also performed computations for mirror geometries obtained by increasing or decreasing all hole diameters by $10 \mathrm{~nm}$, while keeping unchanged the center-to-center hole distances. The results, shown with the dashed curves in Fig. 3, clearly indicate that to AIP license or copyright; see http://apl.aip.org/apl/copyright.jsp 


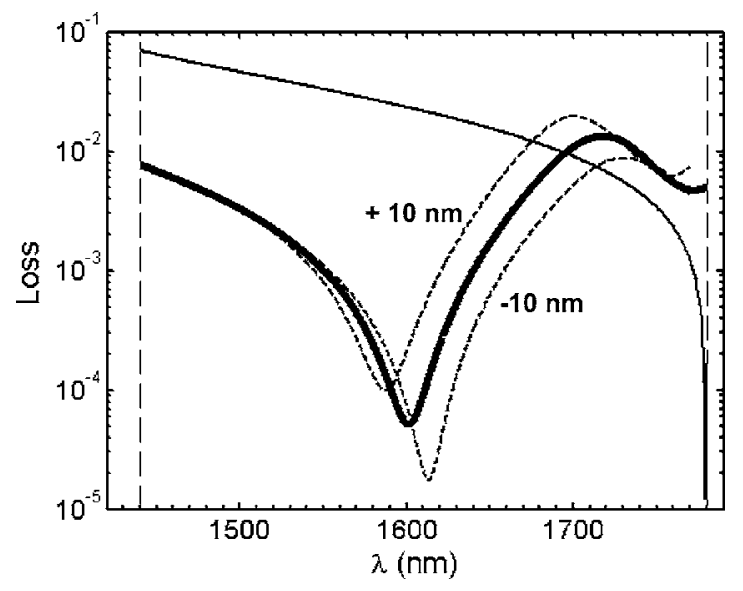

FIG. 3. Beneficial impact of the tapering process on the mirror performance. The bold (thin) solid curves correspond to calculated radiation losses (defined as $\left.1-R_{\infty}\right)$ for tapered (periodic) semi-infinite mirrors $(N=\infty)$. The loss spectra are calculated from dimensions measured on the actual devices, as given in the caption of Fig. 1. The thin-dashed curves are obtained for mirrors with $a \pm 10 \mathrm{~nm}$ variation of all hole diameters, and the vertical dashed lines indicate the band edges of the periodic mirror.

this uncertainty does not impact the analysis. They also evidence that the tapering process is tolerant to fabrication errors. At midgap frequency $(\lambda \approx 1.6 \mu \mathrm{m})$, the losses of the tapered mirror (solid bold curve) are very small $\approx 5 \times 10^{-5}$, a value 400 times lower than that obtained without taper with the fully periodic mirror (thin-solid curve). We have also performed similar computations for mirrors with a finite number $N$ of holes. From these calculations, the theoretical cavity $Q$ 's are deduced by using a Fabry-Perot model, $Q$ $=\pi /\left(1-R_{N}\right)\left[2\left(L / \lambda_{0}\right) n_{g}-\left(\lambda_{0} / \pi\right)\left(\partial \varphi_{N} / \partial \lambda\right)_{\lambda_{0}}\right]$, where $n_{g}=4.12$ is the group index of the fundamental ridge-waveguide mode, $L$ is the cavity defect length (side-to-side separation distance between the two inner holes), and $\varphi_{N}$ is the phase of the modal reflectivity coefficient. For $\lambda=1.6 \mu \mathrm{m}, Q$ $=19.6 /\left(1-R_{N}\right)$, and we find that the measured $Q$ of 8900 for the cavity with $N=4$ corresponds to a finesse $F=\pi /\left(1-R_{N}\right)$ equal to 1400. The dots in Fig. 2(c) represent the $Q$ values computed with the Fabry-Perot model. An excellent agreement with the experimental data (squares) is seen. We have also calculated the cavity mode volume with the Fourier modal method. We found that the mode volume $V$, defined by $V=\int \varepsilon|E|^{2} d V / \max \left(\varepsilon|E|^{2}\right)$ ( $E$ being the electric field of the cavity mode), is equal to $0.62(\lambda / n)^{3}$. Such a small value is due to the strong TIR transversal confinement and, longitudinally, to the taper-design strategy which relies only on the use of mirrorlike nonpropagating Bloch modes in the taper, thus not only the taper implements a transverse-mode-profile conversion but it also reflects light. ${ }^{12}$

The peak transmission $T_{\max }$ at resonance is another important figure of merit. For a Fabry-Perot cavity, one expects that $T_{\max }=(1+L / T)^{-2}$, where $T$ is the mirror in-plane modal transmission, and $L=1-R-T$ is the mirror loss (radiation into the cladding in the present case). The measurement of $T_{\max }$ is critical. We have recorded several times the transmission spectrum of the reference waveguides and that of the $N=4$ cavity, systematically realigning the microlensed fiber with the waveguide input facets before recording. From these spectra, we could not infer the actual $T_{\max }$ value but we could derive a lower bound, $T_{\max }>0.6$ (the theoretical $T_{\max }$ value is 0.99 at $\lambda=1.6 \mu \mathrm{m}$ ). The prediction of a high $T_{\max }$ value for the $N=4$ cavity is consistent with other measurements performed for cavities with $N=2$ and 3 , since we do not observe any significant decrease of the peak transmissions as $N$ increases, the slight fluctuations being well within the uncertainty of the repeatability of the injection into the cleaved facet. In other words, the mode lifetime of the $N$ $=4$ cavity is likely to be limited by in plane transmission rather than radiation loss $(T>L)$. From the Fabry-Perot model, a $T_{\max }$ value larger than 0.6 implies that $T>3.4 L$. Thus the intrinsic (unloaded) $Q$ factor of the cavity, determined by removing the coupling between the waveguide and the cavity $(N=\infty)$, is expected to be larger than 40000 , a value ten times smaller than the theoretical prediction shown in Fig. 2(c).

In summary, ultrashort and highly efficient Bragg mirrors incorporating taper sections have been fabricated in ridge SOI waveguides and have been characterized at telecommunications wavelengths. Microcavities formed by the association of two identical mirrors have been studied. A $Q$ factor of 8900 has been measured for a cavity with a mode volume of $0.62(\lambda / n)^{3}$. The measured $Q$ value is the highest reported so far for cavities (with comparable small mode volumes) manufactured on a substrate. Due to their large finesses, these cavity geometries may be useful for different applications, including Si electro-optic modulators ${ }^{16}$ or filters for wavelength divison multiplexing applications.

CEA-LETI is acknowledged for the access to the micro/ nanofabrication facilities. This work is supported under the project Miraman PNANO06-0215. One of the authors (P.V.) acknowledges support from CNRS and CEA for his Ph.D. Fellowship.

${ }^{1}$ K. J. Vahala, Nature (London) 424, 839 (2003).

${ }^{2}$ B. E. Little, Opt. Photonics News, November 2000, p. 24.

${ }^{3}$ J. Poon, L. Zhu, G. DeRose, and A. Yariv, Opt. Lett. 31, 456 (2006).

${ }^{4}$ A. Melloni and M. Martinelli, J. Lightwave Technol. 20, 296 (2002).

${ }^{5}$ Y. Tanaka, T. Asano, R. Hatsuta, and S. Noda, Appl. Phys. Lett. 88, 011112 (2006).

${ }^{6}$ R. Herrmann, T. Sünner, T. Hein, A. Löffler, M. Kamp, and A. Forchel, Opt. Lett. 31, 1229 (2006).

${ }^{7}$ Y. Tanaka, T. Asano, R. Hatsuta, and S. Noda, Appl. Phys. Lett. 88, 011112 (2006).

${ }^{8}$ J. P. Zhang, D. Y. Chu, S. L. Wu, W. G. Bi, R. C. Tiberio, R. M. Joseph, A. Taflove, C. W. Tu, and S. T. Ho, IEEE Photonics Technol. Lett. 8, 491 (1996).

${ }^{9}$ D. J. Ripin, K. Y. Lim, G. S. Petrich, P. R. Villeneuve, S. Fan, E. R. Thoen, J. D. Joannopoulos, E. P. Ippen, and L. A. Kolodziejski, J. Appl. Phys. 87, $1578(2000)$.

${ }^{10}$ A. S. Jugessur, P. Pottier, and R. M. De La Rue, Electron. Lett. 39, 367 (2003).

${ }^{11}$ P. Lalanne and J. P. Hugonin, IEEE J. Quantum Electron. 39, 1430 (2003).

${ }^{12}$ C. Sauvan, G. Lecamp, P. Lalanne, and J. P. Hugonin, Opt. Express 13, 245 (2005).

${ }^{13}$ S. Lardenois, D. Pascal, L. Vivien, E. Cassan, S. Laval, R. Orobtchouk, M. Heitzmann, N. Bouzaida, and L. Mollard, Opt. Lett. 28, 1150 (2003).

${ }^{14}$ Y. A. Vlasov and S. J. McNab, Opt. Express 12, 1622 (2004).

${ }^{15}$ E. Silberstein, P. Lalanne, J. P. Hugonin, and Q. Cao, J. Opt. Soc. Am. A 18, 2865 (2001).

${ }^{16}$ Q. Xu, B. Schmidt, S. Pradhan, and M. Lipson, Nature (London) 435, 325 (2005). 